\title{
AN INTRODUCTION TO GROUP REPRESENTATIONS AND ORTHOGONAL POLYNOMIALS
}

\author{
Dennis Stanton*
}

\begin{abstract}
An elementary non-technical introduction to group representations and orthogonal polynomials is given. Orthogonality relations for the spherical functions for the rotation groups in Euclidean space (ultraspherical polynomials), and the matrix elements of $S U(2)$ (Jacobi polynomials) are discussed. A general theory for finite groups acting on graphs, giving a finite set of discrete orthogonal polynomials is given. Explicit examples include graphs giving the Krawtchouk and Hahn polynomials.
\end{abstract}

\section{Introduction.}

The purpose of this paper is to present a friendly, non-technical introduction to group representations and orthogonal polynomials. No previous knowledge of group representations is assumed, but a familiarity with orthogonal polynomials is assumed. In particular, this paper emphasizes the classical orthogonal polynomials and their relationship to groups. Other classical special functions can also be studied in this way, e.g. Vilenkin [19] or Miller [14] (which is more elementary). §I of this paper could be considered as a short introduction to the sections of those books relevant to orthogonal polynomials. More modern work on continuous groups and the related analysis has been done by Koornwinder [11] and Dunkl [9]. Some very recent work concerns orthogonal polynomials in several variables [13].

It was not realized until the early 1970's that finite groups could be related to classical orthogonal polynomials. The pioneering work was done by Dunkl [8],[9] and Delsarte [5],[6],[7]. §II is an introduction to the general theory of finite groups. This theory can be generalized to association schemes, which consider relations on a finite set with certain properties. An extensive theory of association schemes can be found in [3] and [5]. A very elementary introduction is given in [16]. A survey of recent work and important problems is given in [4], and in Bannai's paper [2] in this volume.

\section{Notation.}

The classical orthogonal polynomials can be expressed as hypergeometric series. We will use the usual notation for these series (see [15]). We will be most concerned about three sets of polynomials: Jacobi, Krawtchouk and Hahn.

The Jacobi polynomials

$$
P_{n}^{(\alpha, \beta)}(x)=\frac{(\alpha+1)_{n}}{n !}{ }_{2} F_{1}\left(\begin{array}{cc}
-n, & n+\alpha+\beta+1 ; \\
\alpha+1 & \frac{1-x}{2}
\end{array}\right)
$$

*School of Mathematics, University of Minnesota, Minneapolis, MN 55455. This work was partially supported by NSF grant DMS:8700995. 
are orthogonal on $[-1,1]$ with respect to the weight $w(x)=(1-x)^{\alpha}(1-x)^{\beta}$, $\alpha, \beta>-1$. The ultraspherical (or Gegenbauer) polynomials are the special case $\alpha=\beta$ of the Jacobi polynomials.

The Krawtchouk polynomials

$$
K_{n}(x, p, N)={ }_{2} F_{1}\left(\begin{array}{rrr}
-n, & -x ; & 1 \\
& -N & \bar{p}
\end{array}\right)
$$

are orthogonal on the finite set $x=0,1, \cdots, N$ with respect to the binomial distribution $w(x)=\left(\begin{array}{c}N \\ x\end{array}\right) p^{x}(1-p)^{N-x}, 0<p<1$. We will consider $p=1 / 2$, and more generally $p=k /(k+1)$ for a positive integer $k$.

The Hahn polynomials

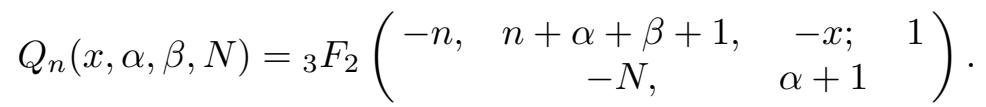

are orthogonal on the finite set $x=0,1, \cdots, N$ with respect to the hypergeometric distribution $w(x)=\left(\begin{array}{c}\alpha+x \\ x\end{array}\right)\left(\begin{array}{c}\beta+N-x \\ N-x\end{array}\right)$. For a positive weight function, $\alpha, \beta>-1$ or $\alpha, \beta<-N$. We shall be considering the second case.

\section{I.1 Continuous groups.}

We begin by considering an elementary problem for polynomials: which polynomials $p(x, y)$ in $x$ and $y$ are harmonic. This means that the Laplacian $\Delta=$ $\partial^{2} / \partial x^{2}+\partial^{2} / \partial y^{2}$ annihilates $p, \Delta p=0$. We can assume that that $p$ is homogeneous of degree $n$, for some $n$ :

$$
p(x, y)=\sum_{i=0}^{n} a_{i} x^{i} y^{n-i}
$$

Clearly $\Delta p=0$ is equivalent to

$$
(i+2)(i+1) a_{i+2}+(n-i)(n-i-1) a_{i}=0 \text { for } 0 \leq i \leq n-2 .
$$

Thus we see that $a_{0}$ and $a_{1}$ are arbitrary and

$$
a_{2 i}=(-1)^{i}\left(\begin{array}{c}
n \\
2 i
\end{array}\right) a_{0}
$$

and

$$
a_{2 i+1}=(-1)^{i}\left(\begin{array}{c}
n \\
2 i
\end{array}\right) a_{1}
$$

Thus an arbitrary harmonic polynomial, homogeneous of degree $n$ in $x$ and $y$, has the form

$$
p(x, y)=A\left[(x+i y)^{n}+(x-i y)^{n}\right]+B i\left[(x+i y)^{n}-(x-i y)^{n}\right],
$$

for arbitrary real constants $A$ and $B$.

In polar coordinates, the two independent solutions in $(2)$ are $r^{n} \cos (n \theta)$ and $r^{n} \sin (n \theta)$. Thus we see the Chebyshev polynomials

$$
T_{n}(\cos \theta)=\cos (n \theta)
$$


and

$$
U_{n-1}(\cos \theta)=\frac{\sin (n \theta)}{\sin (\theta)}
$$

appearing in the solutions to our harmonic polynomial problem. This is not surprising, since $z^{n}$ is a complex analytic function, but what does it have to do with group representations?

Let $O(2)$ be the group of all orthogonal transformations in the $x-y$ plane, so that $O(2)$ consists of all rotations about the origin, and reflections in lines through the origin. The group $O(2)$ acts on the 2-dimensional vector space $V$ of all real harmonic polynomials of degree $n$. This is clear from (2): if a rotation of $\alpha$ is applied, $\theta$ will be increased by $\alpha$, and the addition formula for $\cos (\theta+\alpha)$ will show that $\cos (\theta)$ will be mapped to a linear combination of $\cos (\theta)$ and $\sin (\theta)$. If a reflection through the line $\theta=\beta$ is applied, then $\theta$ will be mapped to $2 \beta-\theta$, and again we use the addition formula to find the appropriate linear combination.

This vector space $V$, together with the action of $G=O(2)$, is an example of an irreducible representation of $G$.

Basically what we have is to "represent" the elements of $G$ as matrices acting on $V$, in a way that preserves the group multiplication, i.e. a product of group elements is represented by a product of matrices. The representation is irreducible because there is no smaller subspace of $V$ which is fixed by $G$. Strictly speaking we have the following definition.

Definition 1. Given a group $G$ and a vector space $V$, an irreducible representation of $G$ is a continuous homomorphism $\phi: G \rightarrow$ Aut $(V)$ such that the only subspaces of $V$ stable under $\phi(G)$ are $V$ and $\{\overrightarrow{0}\}$.

If $V$ is an inner product space over the complex numbers, and $\phi(g)$ preserves the inner product in $V$, then we call $\phi$ a unitary representation. Two repreentations $\phi$ and $\phi^{\prime}$ on $V$ and $V^{\prime}$ are called inequivalent if there is no isomorphism $T: V \rightarrow V^{\prime}$ such that $T \circ \phi(g)=\phi^{\prime}(g) \circ T$ for all $g \in G$.

We can give another example of an irreducible representation of a group, this time a finite group. Let $G$ be the dihedral group of order $2 m$ which acts on a regular $m$-gon in the plane. We assume that the center of the $m$-gon is the origin, and that a vertex lies on the $x$-axis. Consider functions on the vertices of the $m$-gon, whose values at vertex $k$ given by $f_{1}=\cos \left(n \theta_{k}\right)$, where $\theta_{k}=2 \pi k / m, 0 \leq k \leq m-1$. Define $f_{2}$ similarly with sine replacing cosine. These functions are a discrete version of those in (2) and give a 2-dimensional irreducible representation of $G$.

We now turn to orthogonality relations from representations. Since orthogonal polynomials have orthogonality relations, we should see what kinds of relations are available. The next theorem states three possible orthogonality relations, which may or may not be orthogonality relations for orthogonal polynomials.

Orthogonality Theorem. Let $G$ be a compact or finite group, and let $\phi: G \rightarrow$ Aut $(V)$ and $\phi^{\prime}: G \rightarrow A u t\left(V^{\prime}\right)$, be inequivalent unitary irreducible representations on complex vector spaces $V$ and $V^{\prime}$. Let $\left\{v_{1}, \ldots, v_{p}\right\}$ be an orthonormal basis for V. If

$$
T_{i j}^{\phi}(g)=<v_{i}, \phi(g) v_{j}>
$$

is the ij matrix element of $\phi(g)$ with respect to $\left\{v_{1}, \ldots, v_{p}\right\}$, then

(1) $<v_{i}, v_{j}>=\delta_{i j}$ 
(2) $\int_{G} T_{i j}^{\phi}(g) \overline{T_{i^{\prime} j^{\prime}}^{\phi^{\prime}}(g)} d g=\delta_{i i^{\prime}} \delta_{j j^{\prime}} \delta_{\phi \phi^{\prime}} / \operatorname{dim} V$,

(3) $\sum_{j=1}^{p} T_{i j}^{\phi}(g) \overline{T_{k j}^{\phi}(g)}=\delta_{i k}$.

Clearly (1) is just a restatement that is an orthonormal basis for $V$. For a continuous group $G$, if we can parametrize $G$ in some way by real numbers, (2) gives us a chance at absolutely continuous measures for orthogonal polynomials. A finite discrete orthogonality is clearly implied by (3). For (1) for $G=O(2)$, we could define the inner product of our two functions to be integration over the circle. Then the orthogonality is the orthogonality of the two Chebyshev polynomials $T_{n}$ and $U_{n-1}$. The matrix elements for a rotation by $\alpha$ are again trigonometric functions, and integrating over $\alpha$ again gives this orthogonality. The 2 by 2 matrices are easily seen to have (3). We will explicitly find matrix elements for $G=S U(2)$ in $\S I .3$, and interpret the orthogonalities (2) and (3).

\section{I.2 Spherical functions.}

In this section we give the basic properties of spherical functions, also called zonal spherical functions. There is a great literature on the harmonic analysis of these functions. One example of these functions will be the ultraspherical polynomials.

Suppose $G$ acts transitively on a set $X$, as $O(2)$ acts on the circle $S^{1}$ in the plane. Then we have a representation of $G$ on $L^{2}(X)$

$$
L^{2}(X)=\underset{\mu}{\oplus} m_{\mu} V_{\mu}
$$

This representation may not be irreducible, thus it decomposes into many irreducible components, some of which may be equivalent. This means that up to a non-singular transformation between these subspaces, the action of $G$ is identical. The integer $m_{\mu}$ is the multiplicity of the representation $V_{\mu}$ in $L^{2}(X)$. Thus it is assumed in (1), that different $\mu$ correspond to inequivalent representations $V_{\mu}$.

The multiplicities $m_{\mu}$ can be found in the following way. Fix $x_{0} \in X$, and let $K=\left\{g \in G: g\left(x_{0}\right)=x_{0}\right\}$, so that $X=G / K$. The Frobenius reciprocity theorem gives the following property of $m_{\mu}$ and $V_{\mu}$.

Proposition. The subspace of $V_{\mu}$ invariant under $K$ has dimension $m_{\mu}$.

If each $m_{\mu}=1$, we call the representation multiplicity free. In this case each $V_{\mu}$ contains a unique (up to a multiple) vector invariant under $K$. This suitably normalized vector $\phi_{\mu} \in V_{\mu}$, is the spherical function for $V_{\mu} . \phi_{\mu}$ is constant on the $K$-orbits of $G / K$, thus constant on the double cosets $K \backslash G / K$. Since these functions are in distinct representations, they are orthogonal by Orthogonality Theorem (2). Thus, if the double cosets can be parametrized by a single real parameter, we will have an absolutely continuous measure for which the spherical functions are orthogonal. Many of the properties of spherical functions can be found in [9]. We mention here that there is (1) an integral representation using the character of the representation, and (2) an integral product formula.

Let $G=O(2), X=S^{1}, x_{0}=(1,0)$, and $K=<$ the reflection in the $x$-axis $>$. Then the $K$-orbits on $X$ are pairs of points on the unit circle which are complex conjugates. Thus, any function invariant under $K$ on the circle can be considered a function of $\cos (\theta)$. For the irreducible representation of harmonic polynomials of degree $m$ in $\S 2$, the spherical function clearly is the Chebyshev polynomial 
$T_{m}(\cos (\theta))=\cos (m \theta)$. The orthogonality of these polynomials is precisely the orthogonality of spherical functions in distinct representations,

$$
\begin{gathered}
\int_{X} \phi_{\mu}(x) \overline{\phi_{\mu^{\prime}}(x)} d x=0, \mu \neq \mu^{\prime}, \\
\int_{0}^{\pi} T_{m}(\cos (\theta)) \overline{T_{m^{\prime}}(\cos (\theta))} d \theta=0, m \neq m^{\prime} .
\end{gathered}
$$

For the dihedral group acting on an $n$-gon ( $n$ odd) the orthogonality is

$$
\frac{1}{n}+\frac{2}{n} \sum_{k=1}^{n / 2} T_{m}\left(\cos \left(\theta_{k}\right)\right) T_{m^{\prime}}\left(\cos \left(\theta_{k}\right)\right)=0, m \neq m^{\prime} .
$$

Clearly, as $n \rightarrow \infty,(3) \rightarrow(2)$.

For the rotation group $S O(3)$ on the sphere $S^{2}$, one can choose $x_{0}$ to be the north pole, and thus $K$ is the subgroup of rotations about the diameter which passes through $x_{0}$. Then any function $f(x)$ on $S^{2}$, invariant under $K$, only depends upon the angle $\theta=x \cdot x_{0}$. Precisely the same statement can be said about the higher dimensional spheres $S^{n-1}=S O(n) / S O(n-1)$.

Theorem 1. Let Harm $(m)$ be the vector space of harmonic polynomials which are homogeneous of degree $m$. Let $\left.\operatorname{Harm}(m)\right|_{S^{n-1}}$ be the restriction of these functions to the sphere $S^{n-1}$. Then

(1) $L^{2}\left(S^{n-1}\right)=\left.\oplus_{m=0}^{\infty} \operatorname{Harm}(m)\right|_{S^{n-1}}$,

(2) $\left.\operatorname{Harm}(m)\right|_{S^{n-1}}$ is inequivalent to $\left.\operatorname{Harm}\left(m^{\prime}\right)\right|_{S^{n-1}}$, for $m \neq m^{\prime}$,

(3) the spherical function for Harm $\left.(m)\right|_{S^{n-1}}$ is the ultraspherical polynomial $P_{m}^{((n-3) / 2,(n-3) / 2)}(\cos \theta)$, where $x \cdot(0, \ldots, 0,1)=\cos \theta$.

Why should the spherical function be an ultraspherical polynomial? It is easy to see that it must be a polynomial of degree $m$ in $\cos \theta$. The spherical Laplacian $\Delta_{S}$ commutes with the action of $S O(n)$, and so must be a constant on $\left.\operatorname{Harm}(m)\right|_{S^{n-1}}$. The resulting eigenvalue equation is just the differential equation for the ultraspherical polynomials.

Why should (1) hold in Theorem 1? The vector space of homogeneous polynomials of degree $m, \operatorname{Hom}(m)$, decomposes by (see [19, p. 444])

$$
\operatorname{Hom}(m)=\operatorname{Harm}(m) \oplus r^{2} \operatorname{Hom}(m-2) .
$$

Iterating (4) gives

$$
\operatorname{Hom}(m)=\operatorname{Harm}(m) \oplus r^{2} \operatorname{Harm}(m-2) \oplus \cdots .
$$

Thus the vector space of all polynomial functions on $S^{n-1}$ is the vector space of all harmonic polynomial functions.

We can also find $\operatorname{dim}(\operatorname{Harm}(m))$ from $(4)$. There are $\left(\begin{array}{c}n+m-1 \\ n-1\end{array}\right)$ distinct monomials in $n$ variables of degree $m$. This yields

$$
\operatorname{dim}(\operatorname{Harm}(m))=\left(\begin{array}{c}
n+m-1 \\
n-1
\end{array}\right)-\left(\begin{array}{c}
n+m-3 \\
n-1
\end{array}\right) .
$$


Note that for $n=2, \operatorname{dim}(\operatorname{Harm}(m))=2$, as it is in $\S 2$. Also note that every restriction of a non-zero homogeneous polynomial is a non-zero function on $S^{n-1}$.

Finally we come to the invariant measure for the sphere. On $S^{2}$ it is $\sin (\theta) d \theta$, which is $\sin (\theta)$ times the invariant measure for the circle $S^{1}$. It is clear from the Pythagorean theorem, that each dimension gives another factor of $\sin (\theta)$, so that the measure for $S^{n-1}$ is $\sin ^{n-2}(\theta)$. This completes the group theoretic proof that $P_{m}^{((n-3) / 2,(n-3) / 2)}(\cos (\theta))$ are orthogonal on $[0, \pi]$ with respect to $\sin ^{n-2}(\theta)$.

\section{I.3 Matrix elements.}

In this section we compute the matrix elements for $S U(2)$ and show that they are effectively Jacobi polynomials. From the Orthogonality Theorem and the explicit invariant measure for $S U(2)$, we then have the orthogonality for these polynomials.

We let elements of $g \in S U(2)$ be written as

$$
g=\left(\begin{array}{cc}
\alpha & \beta \\
-\bar{\beta} & \bar{\alpha}
\end{array}\right)
$$

where $\alpha$ and $\beta$ are complex numbers satisfying $|\alpha|^{2}+|\beta|^{2}=1$.

Let $V_{m}$ be the vector space of homogeneous polynomials $p(x, y)$ in $x$ and $y$ of degree $m$. The element $g \in S U(2)$ acts by a linear change of variable,

$$
p(x, y) g=p(\alpha x-\bar{\beta} y, \beta x+\bar{\alpha} y) .
$$

We need an appropriate basis for $V$, and a way of parametrizing the group elements of $S U(2)$. It turns out that appropriate multiples of the monomials give an orthonormal basis, on which the group acts unitarily:

$$
v_{i}=c_{i} x^{m / 2-i} y^{m / 2+i},-m / 2 \leq i \leq m / 2 .
$$

(For odd $m, i$ will be half-integral. This slightly unusual way of writing the exponents will simplify the following calculation.) Thus

$$
v_{i} g=c_{i}(\alpha x-\bar{\beta} y)^{m / 2-i}(\beta x+\bar{\alpha} y)^{m / 2+i} .
$$

Expanding by the binomial theorem we find

$$
v_{i} g=\sum_{s=0}^{m / 2-i} \sum_{t=0}^{m / 2+i} c_{i}\left(\begin{array}{c}
m / 2-i \\
s
\end{array}\right)\left(\begin{array}{c}
m / 2+i \\
t
\end{array}\right) \alpha^{m / 2-i-s}(-\bar{\beta})^{s} \bar{\alpha}^{t} \beta^{m / 2+i-t} .
$$

The coefficient of $c_{j} v_{j}$ in (1) is a single sum which is a ${ }_{2} F_{1}$. This is the basic reason that Jacobi polynomials are the matrix elements.

The group elements $g$ can be parametrized by three parameters $(\phi, \psi, \theta)$, called the Euler angles of $S U(2) . \phi$ and $\psi$ are related to the phase of $\alpha$ and $\beta$, and $\theta$ gives the modulus of $\alpha$ (and thus $\beta$ )

$$
g(\phi, \psi, \theta)=\left(\begin{array}{cc}
e^{i(\phi+\psi) / 2} \cos (\theta / 2) & i e^{i(\phi-\psi) / 2} \sin (\theta / 2) \\
i e^{i(-\phi+\psi) / 2} \sin (\theta / 2) & e^{i(-\phi-\psi) / 2} \sin (\theta / 2)
\end{array}\right)
$$

A short calculation gives

$$
\begin{aligned}
T_{i j}^{m}(\phi, \psi, \theta)=\mathrm{constant} & \cdot(1-\cos (\theta))^{(i-j) / 2} \cdot(1+\cos (\theta))^{(i+j) / 2} \\
& e^{-i(i \phi+j \psi)} \cdot P_{m / 2-i}^{(i-j, i+j)}(\cos (\theta)) .
\end{aligned}
$$


The invariant measure on $S U(2)$ in terms of the Euler angles is

$$
d g=\frac{d \phi}{2 \pi} \frac{d \psi}{4 \pi} \frac{\sin (\theta) d \theta}{2} .
$$

Clearly the orthogonality in Orthogonality Theorem (2) is trivial if $i$ or $j$ change. If $i$ and $j$ are fixed, then we have the Jacobi polynomial orthogonality.

What is the discrete orthogonality (3) of the Orthogonality Theorem? It becomes

$$
\begin{aligned}
& \sum_{j=0}^{m}\left(\begin{array}{c}
m \\
j
\end{array}\right)(1-\cos (\theta))^{j-m / 2}(1+\cos (\theta))^{m / 2-j} \\
& \cdot{ }_{2} F_{1}\left(\begin{array}{ccc}
-(m / 2-i), & -j ; & 2 \\
-m & 1-\cos (\theta)
\end{array}\right) \\
& \cdot{ }_{2} F_{1}\left(\begin{array}{ccc}
-(m / 2-k), & -j ; & 2 \\
& -m & 1-\cos (\theta)
\end{array}\right)=0 \text { for } i \neq k \text {. }
\end{aligned}
$$

This is precisely the Krawtchouk polynomial orthogonality with $p=(1-\cos (\theta)) / 2$.

\section{I.4 Clebsch-Gordan coefficients.}

Here we state the basic problem of the Clebsch-Gordan coefficients. These coefficients have orthogonality relations, which turn out to be, for $S U(2)$, the orthogonality relations for Hahn polynomials [1], [12].

The main problem is the following: let $V$ and $W$ be irreducible representations of $G$. Find the decomposition of the tensor product $V \otimes W$.

For $S U(2)$, let $V=V_{j_{1}}$ and $W=V_{j_{2}}$, it can be shown that

$$
V_{j_{1}} \otimes V_{j_{2}}=\oplus_{j=\left|j_{1}-j_{2}\right|}^{j_{1}+j_{2}} V_{j}
$$

(This in fact can be proved from the Chebyshev polynomials of the second kind, which are the characters of $V_{m}$.)

We have two different orthonormal bases for $V \otimes W$ : one made up of tensor products of orthonormal bases from $V=V_{j_{1}}$ and $W=V_{j_{2}}$, and another which consists of the basis vectors for $V_{j}$ inside $V_{j_{1}} \otimes V_{j_{2}}$.

(1) $v_{m_{1}}^{j_{1}} \otimes v_{m_{2}}^{j_{2}},-j_{1} \leq m \leq j_{1},-j_{2} \leq m \leq j_{2}$,

(2) $w_{m}^{j},\left|j_{1}-j_{2}\right| \leq j \leq j_{1}+j_{2},-j \leq m \leq j$.

The Clebsch-Gordan coefficients are the entries of the matrix which accomplishes this change of basis:

$$
v_{m_{1}}^{j_{1}} \otimes v_{m_{2}}^{j_{2}}=\sum_{m, j} C\left(j, m \mid j_{1}, m_{1}, j_{2}, m_{2}\right) w_{m}^{j}
$$

Since each basis is orthonormal, the Clebsch-Gordan matrix must be orthogonal. The discrete orthogonality is equivalent to Hahn polynomial orthogonality.

\section{Finite groups.}

In $\S I$ at no time did we refer to the three term recurrence relation that all orthogonal polynomials satisfy

$$
x p_{i}(x)=A_{i} p_{i+1}(x)+B_{i} p_{i}(x)+C_{i} p_{i-1}(x) .
$$


Given (II.1), it is possible to develop a combinatorial theory of general orthogonal polynomials, [18]. In this theory $p_{n}(x)$ is the generating function for certain weighted lattice paths in the plane, as are the moments $\mu_{n}$ for the measure for $p_{n}(x)$. We cannot, however, interpret a general set of orthogonal polynomials given by (II.1) as the matrix elements of a group, since matrix elements have special properties that general orthogonal polynomials do not have. Nevertheless, we shall see that (II.1) is the key ingredient for orthogonal polynomials on finite groups.

We give the general theory for finite groups in §II.1. These are applied to the Krawtchouk, Hahn and $q$-Hahn polynomials in $\S I I .2, \S I I .3$, and $\S I I .4$. References to combinatorial applications are given in $§ I I .5$.

\section{II.1 Finite groups and graphs.}

There is a complete analogy to the action of the continuous group $S O(n)$ on $S^{n-1}$, and the action of certain finite groups $G$ on certain graphs $X$. In this section we give the general theory of such an analogy, and prove that the spherical functions are always given by orthogonal polynomials. The definitions in $\S I .3$ and the Orthogonality Theorem hold for finite groups.

Recall that a graph $X=(V, E)$ consists of a set of vertices $V$, together with some subcollection $E$ of all 2-element subsets of $V$, called the edges of $X$. Rather than stick to this cumbersome notation, we let $X$ denote the vertices and the graph simultaneously. The metric $d(x, y)$ on $X$ is defined by the length of the shortest path from $x$ to $y$ in $X$. If the graph $X$ is connected, i.e. there is some path between any two vertices, then $d(x, y)<\infty$ for all $x, y \in X$.

Let $G$ be the group of automorphisms of $X$, this means that each $g \in G$ permutes the vertices of $X$ and preserves the edges of $X$. Thus, each $g \in G$ preserves the metric $d$. We assume that $(*)$

$\left({ }^{*} 1\right) X$ is connected,

$\left({ }^{*} 2\right)$ if $d(v, w)=d\left(v^{\prime}, w^{\prime}\right)$, then there exists $g \in G$ such that $(g v, g w)=\left(v^{\prime}, w^{\prime}\right)$.

Under these assumptions $G$ acts transitively on $X$, so that we can think of the action of $G$ on all complex functions on $X, L^{2}(X)$, as comparable to the action of $S O(3)$ on $L^{2}\left(S^{2}\right)$. This action of $G$ on $L^{2}(X)$ is called a permutation representation since $G$ permutes the points of $X$. One example of such a graph is the $n$-gon, whose automorphism group is the dihedral group of order $2 n$. The following theorem shows the spherical functions of $\S I .3$ are applicable.

Theorem 1. The permutation representation of $G$ on $X$ is multiplicity free.

We will prove Theorem 1 while computing the spherical function for each irreducible representation. To find these subspaces of $L^{2}(X)$, we need $|X| \times|X|$ matrices which are indexed by $X$. We let $d$ be the maximum distance in the graph $X$, and define a matrix $A_{i}$ for each $0 \leq i \leq d$ by

$$
\left(A_{i}\right)_{x y}=\left\{\begin{array}{l}
1 \text { if } d(x, y)=i \\
0 \text { otherwise }
\end{array}\right.
$$

The three-term recurrence relation (II.(1)) is given by the multiplication of $A_{1} A_{i}$.

Proposition 1. For any $0 \leq i \leq d$,

$$
A_{1} A_{i}=\alpha_{i} A_{i+1}+\beta_{i} A_{i}+\gamma_{i} A_{i-1},
$$


where, for a fixed $(v, w) \in X \times X$

$$
\begin{aligned}
\alpha_{i} & =|\{z \in X: d(z, v)=1, d(z, w)=i\}|, d(v, w)=i+1 \\
\beta_{i} & =|\{z \in X: d(z, v)=1, d(z, w)=i\}|, d(v, w)=i \\
\gamma_{i} & =|\{z \in X: d(z, v)=1, d(z, w)=i\}|, d(v, w)=i-1
\end{aligned}
$$

Proof. Find the $v w$ entry of each side by counting the appropriate number of triangles in the graph. By the triangle inequality, the three terms listed are the only ones that contribute.

From Proposition 1, we see that $A_{i}=p_{i}\left(A_{1}\right)$, for a finite orthogonal polynomial sequence $p_{i}$. Since each $A_{i}$ is polynomial in $A_{1}$, the algebra of symmetric matrices generated by $\left\{A_{0}, A_{1}, \ldots, A_{d}\right\}$ is commutative. Thus they are simultaneously diagonalizable, and if $\left\{\lambda_{0}>\lambda_{1}>\cdots>\lambda_{d}\right\}$ are the distinct eigenvalues of $A_{1}$, then $\left\{p_{i}\left(\lambda_{0}\right), p_{i}\left(\lambda_{1}\right), \ldots, p_{i}\left(\lambda_{d}\right)\right\}$ are the eigenvalues of $A_{i}$.

It was not an accident that there are $d+1$ distinct eigenvalues of $A_{1}$. The dimension of the algebra of matrices generated by $\left\{A_{i}: 0 \leq i \leq d\right\}$ is clearly $d+1$. This algebra is a polynomial algebra in $A_{1}$, thus $A_{1}$ has $d+1$ distinct eigenvalues.

Let $V_{0}, V_{1}, \ldots, V_{d}$ be the eigenspaces for $A_{1}$ corresponding to $\left\{\lambda_{0}>\lambda_{1}>\cdots>\right.$ $\left.\lambda_{d}\right\}$. These will be the irreducible representations we seek. Thus we need to find how the spherical functions are related to the polynomials $p_{i}(x)$, and what kind of orthogonality relation they have. To do this, we will use the projection matrices $P r_{0}, P r_{1}, \ldots, P r_{d}$, where $P r_{i}$ is the projection of $L^{2}(X)$ onto $V_{i}$. By definition we have

$$
A_{i}=\sum_{j=0}^{d} p_{i}\left(\lambda_{j}\right) P r_{j}
$$

which we can invert to

$$
\operatorname{Pr}_{j}=\sum_{i=0}^{d} q_{j}(i) A_{i} .
$$

We use the orthogonality $\operatorname{Pr}_{j} P r_{k}=P r_{j} \delta_{j k}$, by expanding each $P r_{j}$ and $P r_{k}$ in terms of the $A_{i}$ 's, then multiplying the $A_{i}$ by counting triangles in the graph of given side lengths (call this number $c_{i s}^{t}$ ), and equating coefficients of $A_{l}$. The result is

$$
q_{j}(l) \delta_{j k}=\sum_{i, s} q_{j}(i) q_{k}(s) c_{i s}^{l}
$$

Even though (3) is a double sum, it can easily reduce to a single sum. If $l=0$, then clearly $c_{i s}^{0}=0$ unless $i=s$. So we have

$$
q_{j}(0) \delta_{j k}=\sum_{i} q_{j}(i) q_{k}(i) c_{i i}^{0}
$$

This is an orthogonality relation for $q_{j}(i)$, with weights $c_{i i}^{0}$, the sizes of a sphere of radius $i$ centered at any point of the graph. If $q_{j}(i)$ is a polynomial of degree $j$ 
evaluated at some $\mu_{i}$, then we call the graph $Q$-polynomial (see [2]). It is already $P$-polynomial.

We now have two orthogonality relations for $q_{j}(i)$ : (4) and the definition (2) of $q_{j}(i)$ as the inverse matrix to $p_{i}\left(\lambda_{j}\right)$. Thus, up to constants (which can be explicitly found), we can say that these two quantities are equal. The resulting orthogonality for $p_{i}\left(\lambda_{j}\right)$ is

$$
\frac{1}{|X|} \sum_{j=0}^{d} p_{k}\left(\lambda_{j}\right) p_{i}\left(\lambda_{j}\right) \operatorname{dim} V_{j}=\delta_{i k} c_{k k}^{0} .
$$

We also see that the multiplication formula for $A_{i} A_{j}$ gives the following linearization formula for the polynomials $p_{i}(x)$ :

$$
p_{i}\left(\lambda_{m}\right) p_{j}\left(\lambda_{m}\right)=\sum_{k} c_{i j}^{k} p_{k}\left(\lambda_{m}\right)
$$

If $x_{0} \in X$ is fixed, and $K$ is the stablizer of $x_{0}$, then any function on $X$ invariant under $K$ must be constant on the $K$ orbits on $X$. They are

$$
\Omega_{j}=\left\{x \in X: d\left(x, x_{0}\right)=j\right\} .
$$

Thus the orthogonality relation for spherical functions will be

$$
\sum_{j=0}^{d} \phi_{m}\left(\Omega_{j}\right) \phi_{m^{\prime}}\left(\Omega_{j}\right)\left|\Omega_{j}\right|=0 \text { if } m \neq m^{\prime} .
$$

This appears to agree with (5). Thus the spherical functions $\phi_{m}\left(\Omega_{j}\right)$ should be $p_{j}\left(\lambda_{m}\right)$. This is true, and can be verified from the eigenvalue equation

$$
\left(A_{1} \phi\right)\left(\Omega_{j}\right)=\lambda_{m} \phi\left(\Omega_{j}\right) .
$$

(6) becomes precisely the three-term recurrence relation (II.(1)), and shows that $\phi_{m}\left(\Omega_{j}\right)$ is a polynomial of degree $j$ in $\lambda_{m}$. This proves that each $V_{m}$ has a unique spherical function, so is irreducible by the Proposition in $§ I .2$. We summarize these conclusions in the next theorem.

Theorem 2. Let $X$ be a graph satisfying $\left({ }^{*}\right)$ of maximum distance $d$. Then the permutation representation of $G$ on $X$ is multiplicity free, $L^{2}(X)=\underset{m=0}{\oplus} V_{m}$, where $V_{m}$ is the mth eigenspace of the adjacency matrix of $X$. Moreover, the spherical function $\phi_{m}(x) \in V_{m}$ is given by $\phi_{m}(x)=p_{j}\left(\lambda_{m}\right), d\left(x, x_{0}\right)=j$, for a finite orthogonal polynomial sequence $p_{j}(x)$.

The analogies between the finite case $X$ and the classical case $S^{n-1}$ are summarized in the following list as cases (a) and (b).

(1a) Any differential operator commuting with $S O(n)$ is a polynomial in the spherical Laplacian $\Delta_{S}$.

(1b) Any operator commuting with $G$ is a polynomial in $A_{1}-\left|\Omega_{1}\right| I$.

(2a) The eigenvalues of $\Delta_{S}$ are non-positive.

(2b) The eigenvalues of $A_{1}-\left|\Omega_{1}\right| I$ are non-positive. 
(3a) $\Delta_{S} f=\lambda f$ is a second order differential equation for $f$.

(3b) $A_{1} f=\lambda f$ is a second order difference equation for $f$.

(4a) For $f \in \operatorname{Harm}(k)$, the mean value operator $T_{\theta}$ satisfies $T_{\theta} f=p_{k}(\cos (\theta)) f$.

(4b) For $f \in V_{k}$, the mean value operator $A_{j}$ satisfies $A_{j} f=p_{j}\left(\lambda_{k}\right) f$.

(5a) A limit of the mean value value operator, $\lim _{\theta \rightarrow 0} T_{\theta}-I=c \Delta_{S}$.

(5b) A difference of the mean value operator is $A_{1}-\left|\Omega_{1}\right| I$ is the "closest" difference operator.

\section{II.2 Krawtchouk polynomials.}

We next give an appropriate graph $X$, find the eigenspaces $V_{j}$, realize the Krawtchouk polynomials $K_{j}(x, 1 / 2, d)$ as spherical functions.

Let $X$ be the $d$-dimensional cube: the vertices of $X$ are all $2^{d} d$-tuples of 0 's and 1's, and two vertices are connected by an edge if they agree in all but one coordinate. The distance between two vertices is the number of coordinates in which they differ. The group $G$ which acts on $X$ is the hyperoctahedral group: all $d$ ! permutations of the coordinates, and all $2^{d}$ interchanges of 0's and 1's. This group is a semidirect product of the symmetric group $S_{d}$ and a product of $d$ cyclic groups of order 2 . The conditions $(*)$ are easily verified.

First we find the three-term recurrence relation of Proposition 1. Let $x_{0}=$ $00 \cdots 0$ be fixed, and let $x=1 \cdots 10 \cdots 0$ be fixed vertex which is distance $i$ from $x_{0}$, so that $x$ has $i 1$ 's. There are $d$ vertices which are distance 1 from $x: i$ of them are distance $i-1$ from $x_{0}$, and $d-i$ of them are distance $i+1$ from $x_{0}$. We find

$$
\lambda p_{i}(\lambda)=i p_{i-1}(\lambda)+(d-i) p_{i+1}(\lambda) .
$$

Equation (1) for $i=d$ determines the eigenvalues $\lambda_{j}=d-2 j$. These can be derived independently by explicitly finding the eigenspaces $V_{j}$. For $1 \leq i \leq d$, let $x_{i}$ be the ith coordinate function on $X$ defined by $x_{i}(0)=-1$, and $x_{i}(1)=1$. Let $V_{j}$ be the span of all square free monomials of degree $j$ in the $x_{i}$ 's. It is easy to see that these monomials are orthogonal, so that $\operatorname{dim} V_{j}=\left(\begin{array}{l}d \\ j\end{array}\right)$. We now verify that

$$
A_{1} x_{1} x_{2} \cdots x_{j}=(d-2 j) x_{1} x_{2} \cdots x_{j},
$$

so that $d-2 j$ is the eigenvalue of $A_{1}$ on $V_{j}$. To see this, evaluate both sides of (2) at an arbitrary vertex $y$ of $X$. If we change one of the first $j$ entries of $y$, then the value of the monomial changes sign. If we change one of the last $d-j$ entries of $y$, the value of the monomial remains the same. So the eigenvalue is $d-2 j$.

Next we find an explicit formula for the spherical functions (the Krawtchouk polynomials) as a ${ }_{2} F_{1}$. What monomial in $V_{j}$ is fixed by the subgroup $K$ of $G$ fixing $x_{0}$ ? Clearly $K=S_{d}$, the symmetric group of the coordinates, so the only polynomial in $V_{j}$ invariant under $S_{d}$ is the elementary symmetric function $e_{j}\left(x_{1}, x_{2}, \ldots, x_{d}\right)$ of degree $d$. We must evaluate this polynomial at a vertex which is distance $i$ from $x_{0}, y=1 \cdots 10 \cdots 0$. The function $e_{j}\left(x_{1}, x_{2}, \ldots, x_{d}\right)$ can be thought of as the sum over all subsets $S$ of $\{1,2, \ldots, d\}$ of size $j$. Let $|S \cap\{1,2, \ldots, i\}=k|$, and $|S \cap\{i+1, i+2, \ldots, d\}=j-k|$. The value of the monomial corresponding to $S$ at $y$ is $(-1)^{j-k}$, so we have

$$
\phi_{j}\left(\Omega_{i}\right)=\text { constant } \cdot \sum_{k=0}^{j}\left(\begin{array}{l}
i \\
k
\end{array}\right)\left(\begin{array}{l}
d-i \\
j-k
\end{array}\right)(-1)^{j-k} .
$$


This is equivalent (by a ${ }_{2} F_{1}$ transformation) to $(\mathrm{Kr})$.

The weight function for $\phi_{j}\left(\Omega_{i}\right)$ is $\left|\Omega_{i}\right|=\left(\begin{array}{l}d \\ i\end{array}\right)=\operatorname{dim} V_{i}$. Note that if the spherical function is normalized to be 1 at $i=0$, it is symmetric under the interchange of $i$ and $j$. This always occurs if the group $G$ is a semidirect product with an abelian normal subgroup.

The Krawtchouk polynomials $K_{j}(i, k /(k+1), d)$ are the spherical functions for the graph of all $d$-tuples of 0 's, 1 's, $\ldots, k$ 's [8].

\section{II.3 Hahn polynomials.}

Next we give an appropriate graph $X$ for the Hahn polynomials.

Let $X$ be the set of all $n$-subsets of $\{1,2, \ldots, v\}$, so that $|X|=\left(\begin{array}{l}v \\ n\end{array}\right)$. two subsets $A$ and $B$ are connected by an edge if $|A \cap B|=n-1$. If we assume that $2 n \leq v$, then the maximum distance is $n$, which occurs if two subsets are disjoint. In general, $d(A, B)=n-|A \cap B|$. The symmetric group $S_{v}$ acts on $X$ and the conditions (*) are satisfied.

First we find the size of the spheres of radius $j$, which are the weights of the spherical functions. Fix $x_{0}=\{1,2, \ldots, n\}$. The number of $n$-subsets $B$ of $\{1,2, \ldots, v\}$ such that $\left|x_{0} \cap B\right|=n-j$ is

$$
\left(\begin{array}{c}
n \\
n-j
\end{array}\right)\left(\begin{array}{c}
v-n \\
j
\end{array}\right)
$$

These are the weights for the Hahn polynomials (see (Ha)) with $\alpha=n-v-1$, $\beta=-n-1$, since then

$$
\left(\begin{array}{c}
\alpha+j \\
j
\end{array}\right)\left(\begin{array}{c}
\beta+n-j \\
n-j
\end{array}\right)=\left(\begin{array}{c}
n \\
n-j
\end{array}\right)\left(\begin{array}{c}
v-n \\
j
\end{array}\right)
$$

The three term recurrence relation is easily found as

$$
\begin{aligned}
\lambda p_{i}(\lambda)=i^{2} p_{i-1}(\lambda)+ & (n-i)(v-n-i) p_{i+1}(\lambda) \\
+ & {\left[n(v-n)-i^{2}-(n-i)(v-n-i)\right] p_{i}(\lambda) . }
\end{aligned}
$$

This is the second order difference equation for Hahn polynomials, or the three term recurrence relation for the dual Hahn polynomials.

To define the eigenspaces $V_{j}, 0 \leq j \leq n$, we define monomial functions on $X$ by

$$
x_{i_{1}} x_{i_{2}} \cdots x_{i_{j}}(A)=\left\{\begin{array}{l}
1 \text { if }\left\{i_{1}, i_{2}, \cdots, i_{j}\right\} \subset A \\
0 \text { otherwise }
\end{array}\right.
$$

(These functions are called sections of the zeta function in the theory of posets.) Let $\operatorname{Hom}(j)$ be the span of all monomials of degree $j$, and let $D=\partial / \partial x_{1}+\cdots+\partial / \partial x_{v}$ be the "down" operator. $G$ acts on $\operatorname{Hom}(j)$, but the action is not irreducible. Since $D$ commutes with the action of $G, G$ also acts on $\operatorname{Harm}(j)=\operatorname{Hom}(j) \cap \operatorname{kerD}$.

Again to show $\operatorname{Harm}(j)=V_{j}$, i.e. $\operatorname{Harm}(j)$ is irreducible, we show that $\operatorname{Harm}(j)$ has a unique spherical function $\phi_{j}$, and compute what $\phi_{j}$ is. The subgroup fixing $x_{0}$ is $K=S_{n} \times S_{v-n}$. This time the $K$-invariant polynomials in $H o m(j)$ are spanned by a product of elementary symmetric functions

$$
e_{s}\left(x_{1}, \cdots, x_{n}\right) e_{j-s}\left(x_{n+1}, \cdots, x_{v}\right), 0 \leq s \leq j
$$


We find which linear combination is annihilated by $D$ using

$$
\begin{aligned}
D e_{s}\left(x_{1}, \cdots, x_{n}\right) & =(n-s+1) e_{s-1}\left(x_{1}, \cdots, x_{n}\right) \\
D e_{j-s}\left(x_{n+1}, \cdots, x_{v}\right) & =(v-n-j+s+1) e_{j-s-1}\left(x_{n+1}, \cdots, x_{v}\right)
\end{aligned}
$$

Suppose

$$
D\left(\sum_{s=0}^{j} c_{s} e_{s}\left(x_{1}, \cdots, x_{n}\right) e_{j-s}\left(x_{n+1}, \cdots, x_{v}\right)\right)=0 .
$$

From (1) we see that $c_{s+1}(-s+n)=(v-n-j+s+1) c_{s}$, so

$$
c_{s}=\frac{(v-n-j+1)_{s}}{(-n)_{s}} c_{0} .
$$

This proves that the $K$-invariant functions in $\operatorname{Harm}(j)$ are 1-dimensional, so $\operatorname{Harm}(j)$ is irreducible.

To evaluate $\phi_{j}\left(\Omega_{i}\right)$, fix $A=\{1, \cdots, n-i, n+1, \cdots, n+i\} \in \Omega_{i}$. To evaluate

$$
e_{s}\left(x_{1}, \cdots, x_{n}\right) e_{j-s}\left(x_{n+1}, \cdots, x_{v}\right)(A)
$$

we count the number of pairs of subsets $(B, C)$, where $|B|=s, B \subset\{1, \cdots, n-i\}$, $|C|=j-s, C \subset\{n+1, \cdots, n+i\}$. This number is $\left(\begin{array}{c}n-i \\ s\end{array}\right)\left(\begin{array}{c}i \\ j-s\end{array}\right)$, so

$$
\phi_{j}\left(\Omega_{i}\right)=\text { constant } \sum_{s=0}^{j} \frac{(v-n-j+1)_{s}}{(-n)_{s}}\left(\begin{array}{c}
n-i \\
s
\end{array}\right)\left(\begin{array}{c}
i \\
j-s
\end{array}\right) .
$$

This ${ }_{3} F_{2}$ is equivalent to (Ha) with $\alpha=n-v-1$ and $\beta=-n-1$ by a ${ }_{3} F_{2}$ transformation. From $i=1$ we find $\lambda_{j}=n(v-n)-j(v+1-j)$.

Finally the $L^{2}$ norm of the polynomials can be found from $\operatorname{dim} V_{j}=\left(\begin{array}{l}v \\ j\end{array}\right)-\left(\begin{array}{c}v \\ j-1\end{array}\right)$.

\section{II.4 $q$-Hahn polynomials.}

Next we give a very short introduction to a $q$-analog of Hahn polynomials, whose explicit formula is in terms of basic hypergeometric series (see Rahman's paper [15] in this volume).

The main idea is that $q$-binomial coefficients

$$
\left[\begin{array}{l}
n \\
k
\end{array}\right]_{q}=\frac{(q ; q)_{n}}{(q ; q)_{k}(q ; q)_{n-k}}
$$

will replace the binomial coefficients that were used in $\S I I .3$. Thus we need to know what $\left[\begin{array}{l}n \\ k\end{array}\right]_{q}$ counts instead of $\left(\begin{array}{l}n \\ k\end{array}\right)$, and how $q$ is involved. The answer is: $\left[\begin{array}{l}n \\ k\end{array}\right]_{q}$ is the number of $k$-dimensional vector spaces of an $n$-dimensional vector space over a finite field of order $q$.

We let $X$ be the set of all $n$-dimensional vector spaces of an $v$-dimensional vector space over a finite field of order $q$. We let the edges of $X$ be pairs of subspaces $A$ - $B$ such that $\operatorname{dim}(A \cap B)=n-1$. The group $G=G L_{v}(q)$ satisfies the $\left(^{*}\right)$ condition. The distance between two subspaces $A$ and $B$ is $d(A, B)=n-\operatorname{dim}(A \cap B)$. How many subspaces are distance $i$ from a fixed subspace? The answer is

$$
\left[\begin{array}{c}
n \\
n-i
\end{array}\right]_{q}\left[\begin{array}{c}
v-n \\
i
\end{array}\right]_{q} q^{i^{2}}
$$

the weights for $q$-Hahn polynomials.

We state, and do not give the details (see [7],[17]) for the calculation of the spherical function. 
Theorem. The spherical function $\phi_{j}\left(\Omega_{i}\right)$ is a q-Hahn polynomial

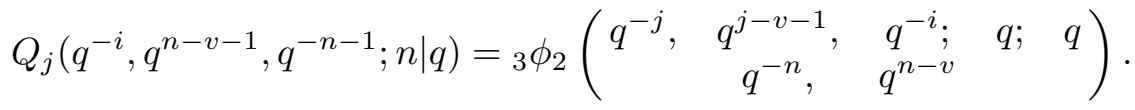

There are also natural $q$-analogs of the Krawtchouk polynomials. These again are related to classical groups over fields of order $q$, whose Lie algebra is of type $B_{n}$, $C_{n}$, and $D_{n}$. Details occur in [17]. All known infinite families, whose maximum distance $d \rightarrow \infty$ are related to these groups (or their Weyl groups). It is believed that this is it [2].

\section{Remarks.}

There are many applications of the discrete orthogonal polynomials in $\S I$ II to coding theory and the theory of designs. The location of the zeros of the polynomials is critical. Bannai's paper [2] in this volume contains many of these results. An example of an analytic theorem motivated by combinatorial constraints is Leonard's theorem. This theorem states that if $p_{i}\left(\lambda_{j}\right)=q_{j}\left(\mu_{i}\right)$ for another finite set of orthogonal polynomials $q_{j}(x)$, then $p_{i}(x)$ must be a special or limiting case of the Askey-Wilson ${ }_{4} \phi_{3}$ polynomials.

The continuous version of the groups and graphs in $\S I I$. are called rank one symmetric spaces of compact type. They have been classified [20], and the spherical functions are all Jacobi polynomials. There is also work on rank $k$ symmetric spaces, and orthogonal polynomials in several variables. Bannai [2] contains references to this work.

An exciting recent area of research is the relationship of quantum groups to qorthogonal polynomials. Koornwinder's paper in this volume [10] surveys the work to this day. The quantum groups are not groups, but algebras with a multiplication that in a sense is dual to group multiplication. For $S U(2)$, the irreducible representations of this algebra are analogous to the polynomial representations in $§$ I.3. Explicit calculations can be made, using a form of the binomial theorem for letters $A$ and $B$ which satisfy $B A=q A B$,

$$
(A+B)^{n}=\sum_{k=0}^{n}\left[\begin{array}{l}
n \\
k
\end{array}\right]_{q} A^{k} B^{n-k}
$$

When the analogous expansion to (I.3(1)) is made, the matrix elements are ${ }_{2} \phi_{1}$ 's instead of ${ }_{2} F_{1}$ 's.

\section{REFERENCES}

1. R. Askey and J. Wilson, A set of orthogonal polynomials that generalize the Racah coefficients or the $6-j$ symbols, SIAM J. Math. Anal. 10 (1979), 1008-1016.

2. E. Bannai, Orthogonal polynomials in coding theory and algebraic combinatorics, this volume.

3. E. Bannai and T. Ito, Algebraic Combinatorics I, Benjamin/Cummins, Menlo Park California, 1984.

4. Current research on algebraic combinatorics, Graphs and Combinatorics 2 (1986), $287-308$.

5. P. Delsarte, An algebraic approach to the association schemes of coding theory, Philips Res. Repts. Supp. 10.

6. $n)-q^{k} F(i, k, n)$, SIAM J. Appl. Math. 31 (1976), 262-270. 
7. _ Hahn polynomials, discrete harmonics, and t-designs, SIAM J. Appl. Math. 34 (1978), 157-166.

8. C. Dunkl, A Krawtchouk polynomial addition theorem and wreath products of symmetric groups, Indiana Math. J. 25 (1975), 335-358.

9. S_ Spherical functions on compact groups and applications to special functions, Symposia Mathematica 22 (1979), 145-161.

10. T. Koornwinder, Orthogonal polynomials and special functions in connection with quantum groups, this volume.

11. Krawtchouk polynomials, a unification of two different group theoretic interpretations, SIAM J. Math. Anal. 13 (1982), 1011-1023.

12. __ Clebsch-Gordan coefficients for SU(2) and Hahn polynomials, Nieuw. Archief Wisk. (3) 29 (1981), 140-155.

13. I. G. Macdonald, Orthogonal polynomials associated with root systems, this volume.

14. W. Miller, Symmetry groups and Their Applications, Academic Press, New York, 1972.

15. M. Rahman, Some extensions of the beta integral and the hypergeometric function, this volume.

16. N. J. A. Sloane, An introduction to association schemes and coding theory, Theory and Application of Special Functions, R. Askey ed., 1975, pp. 225-260.

17. D. Stanton, Orthogonal polynomials and Chevalley groups, Special Functions: Group Theoretic Aspects and Applications, R. Askey et al. (eds.), Reidel, Dordrecht, 1984, pp. 87-128.

18. X. Viennot, Une theorie combinatoire des polynomes orthogonaux generaux, lecture Notes, UQAM, 1983.

19. N. Vilenkin, Special Functions and the Theory of Group Representations, Translations of the AMS, vol. 22, 1968.

20. H. Wang, Two-point homogeneous spaces, Ann. of Math. 55 (1952), 177-191. 